\title{
FIXED-ORDER MIXED NORM DESIGNS FOR BUILDING VIBRATION CONTROL
}

\author{
Mark S. Whorton \\ NASA George C. Marshall Space Flight Center \\ Marshall Space Flight Center, AL 35812 \\ Anthony J. Calise \\ Georgia Institute of Technology \\ Atlanta, GA 30332
}

\begin{abstract}
This study investigates the use of $\mathrm{H}_{2}, \mu$ synthesis, and mixed $\mathrm{H}_{2} / \mu$ methods to construct full order controllers and optimized controllers of fixed dimensions. The benchmark problem definition is first extended to include uncertainty within the controller bandwidth in the form of parametric uncertainty representative of uncertainty in the natural frequencies of the design model. The sensitivity of $\mathrm{H}_{2}$ design to unmodeled dynamics and parametric uncertainty is evaluated for a range of controller levels of authority. Next, $\mu$-synthesis methods are applied to design full order compensators that are robust to both unmodeled dynamics and to parametric uncertainty. Finally, a set of mixed $\mathrm{H}_{2} / \mu$ compensators are designed which are optimized for a fixed compensator dimension. These mixed norm designs recover the $\mathrm{H}_{2}$ design performance levels while providing the same levels of robust stability as the $\mu$ designs. It is shown that designing with the mixed norm approach permits higher levels of controller authority for which the $\mathrm{H}_{2}$ designs are destabilizing. The benchmark problem is that of an active tendon system. The controller designs are all based on the use of acceleration feedback.
\end{abstract}

\begin{abstract}
Introduction
Concepts for active and hybrid active/passive control in building structures have been explored by a number of authors (e.g. Ref. 1). More recent attention has been given to the applicationof robust control theory in the context of $\mathrm{H}_{\infty}$ design (e.g. Refs. 2, Vef $\{$ Yang\}, Vref $\{$ Kose $\}$ ). Robust control is concerned with maintaining performance with uncertainty in the dynamical system. Uncertainties are basically the discrepancies between the mathematical model of the plant to be controlled and the actual plant. It is often the case that the higher modes of vibration of a structure are discarded in the model. Thus one form of uncertainty is due to neglected dynamics. Another example is the mass or stiffness of some element of the dynamical system, which will always differ to some degree from the model value. This is called parametric uncertainty. Exogenous inputs, or disturbances, are also uncertainties. They affect performance, but not stability. For an actively controlled building, seismic activity, wind gusts, and sensor noise are examples of exogenous disturbances. Robust control means having a controller which maintains stability and performance specifications in the presence of uncertainty. Performance is measured by the response of the controlled system to worst case bounded disturbances. Specific definitions of performance will be given later. Before robust control theory was
\end{abstract}


developed, multivariable controller design techniques provided only sufficient conditions for robust performance, which could be very conservative for poorly conditioned plants (or for well conditioned plants with non-round performance specifications). What distinguishes robust control theory is that it provides a systematic approach to evaluating and designing controllers that attempt to maintain performance specifications in the presence of uncertainty in a non- conservative fashion. Because robust controllers can tolerate uncertainties, control of a building structure's seismic response is an ideal application. The examples of uncertainties given above are all present. Also, deformations in the structure will cause changes in the inherent stiffness and passive damping. The nonlinearities encountered in the deformation of the building structure during a seismic event can also be considered as uncertainties.

While robust control provides performance in the presence of uncertainties, the performance is defined by an $\mathrm{H}_{\infty}$ norm measure, which may not be well suited to the performance objectives. In cases such as minimizing control energy, line-of-sight pointing error, or (as in this paper) minimizing the root-mean-square (rms) vibration response of a structure, the $\mathrm{H}_{2}$ norm is a better measure of performance. However, it is well known that $\mathrm{H}_{2}$ design at high control authority levels has very poor robust stability properties. These issues are addressed in the mixed $\mathrm{H}_{2} / \mathrm{H}_{\infty}$ design method. Mixed $\mathrm{H}_{2}$ $/ \mathrm{H}_{\infty}$ design seeks to minimize the $\mathrm{H}_{2}$ norm of one transfer function while satisfying an overbound constraint on the $\mathrm{H}_{\infty}$ norm of another transfer function. Using this approach allows one to design for $\mathrm{H}_{2}$ nominal performance while maintaining the robust stability piovisions of $\mathrm{H}_{\infty}$ design.

The problem from a controls point of view is the need to develop a controller that can reliably accommodate the uncertainty in excitation that is characteristic of earthquakes, while at the same time handle the presence of uncertainties caused by inelastic structural response. The purpose of this paper is to examine design approaches which achieve nominal performance only $\left(\mathrm{H}_{2}\right)$, robust performance ( $\mu$-synthesis), and nominal performance/robust stability (mixed $\mathrm{H}_{2} / \mu$ ), applied to the problem of building structural control. The challenge is to achieve the highest attainable level of rms performance for a specified bounded set of uncertainties. This paper provides a brief description of the $\mathrm{H}_{2}, \mu$-synthesis, and mixed $\mathrm{H}_{2} / \mu$ design methods, emphasizing the role of uncertainty modeling. A comparison of these controller design techniques is given, using the three-story tendon controlled structure at the National Center for Earthquake Engineering Research.

Modeling for Design and Evaluation Design of a high performance control system is inherently dependent on the availability of an accurate design model and knowledge of associated uncertainties. For structural systems such as the building control benchmark problem, models are typically of higher order than is desirable, especially when frequency dependent weights are included in the control design. As in the case of this benchmark problem, the complexity of a control system may have constraints that require either reducing the model dimension for control design, reducing the dimension of the control system, or designing optimal controllers of fixed dimension. This paper implements the latter approach. A reduced order nominal design model will be obtained from the 
evaluation model in Ref. Iref\{Spencer\}. Additionally, a model formulation will be presented which accounts for real parameter uncertainties in the design model.

A six state nominal performance design model was obtained by balancing and residualizing the 20 state evaluation model, retaining the modes at $2.268,7.332$, and $12.240 \mathrm{~Hz}$. Inputs to the generalized plant for $\mathrm{H}_{2}$ control design consist of the ground acceleration disturbance, $\$ \backslash d d o t\{x\}$ g\$, sensor noise, and the tendon control input, $u$. Performance outputs include the weighted displacement of the three floors relative to the ground, zp, and the weighted control force, $z$. The measurement output, $y$, is the absolute acceleration of each of the three floors. All units are in volts.

\section{Robust Performance Design Model}

The nominal evaluation model for the benchmark problem may be extended to include parametric uncertainty within the control bandwidth in the form of errors in the modal damping and frequency squared terms as introduced in Ref. Vref\{Balas1\}.

Uncertainty will only be used for the natural frequency squared terms in this paper, but for completeness, the formulation for uncertain modal damping will also be presented. Although the uncertain natural frequency square terms are real parameters, using a complex uncertainty also accounts for variations in modal damping if a hysteretic damping model is assumed.

In modal form, the nominal A matrix for a second order system is written

Ibegin \{equation\}
A_ $0=V$ eft $[$ begin array $\}\{c c\} 0 \& 111$ lomega^2 \& -2lzetalomega

lend \{array\} Vright] $\|$

lend equation $\}$

Introducing multiplicative uncertainty in the modal frequency square and modal damping terms results in

begin $\{$ eqnarray\}

$A \&=\& V$ eft $[$ begin $\{$ array $\}\{\mathrm{c} c\} 0 \& 11$

- lomega^2(1+ldelta_1) \& -

2betalomega(1+ldelta_2)

lend $\{$ array $\}$ Vright] Vabel $\{$ eq:uncA $\} \|$

$\&=\&$ A_0 + Delta A

lend $\{$ eqnarray $\} \|$

where

Ibegin eqnarray\}

Delta A \& $=\&$ Veft $[$ begin $\{$ array $\}\{c c\} 0$ \& 011

-lomega^2ldelta_1 \& -2lzetalomegaldelta_2

lend \{array\} Vright] 11

$\&=\&$ Idelta_1 Veft[ $\operatorname{lbegin}\{$ array $\}\{c\} 0 \| 1$ lend $\{$ array $\}$ Vright $]$

Veft[-lomega^2 $1 ; ; ; 0$ lright] +

Idelta_2 Veft[ Veegin array $\{$ c $\} 0 \| 1$

lend\{array\} lright]

Veft[0 $; ;$-2lzetalomegalright] 
lend \{eqnarray\}

For a system with $\mathrm{n}$ total modes and $\mathrm{m}$ uncertain modes, S A $=$ A_0 + lsum_ $\{i=1\}^{\wedge}\{m\} \mid$ Delta A_i $\$$ and

Ibegin \{equation\}

Delta $A_{-} \mathrm{i}=\left(\mathrm{e}_{-}\{2 \mathrm{i}\}\right) \backslash$ delta_$\{1 \mathrm{i}\}(-$ lomega $\left.\overline{\mathrm{i}}^{\wedge} 2\right)\left(\mathrm{e}_{-}\{2 \mathrm{i}-1\}\right)^{\wedge} \mathrm{T}$

$+\left(e_{-}\{2 \mathrm{i}\}\right) \backslash \mathrm{delta}\{2 \mathrm{i}\}(-$

2lzeta_ilomega_i) $\left(e_{-}\{2 \mathrm{i}\}\right)^{\wedge} \mathrm{T}$

lend $\{$ equation

where $\$\left(e_{j} j\right) \$$ is the jth standard basis vector

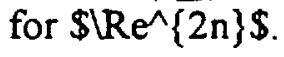

Defining $k$ to be the set of indices of uncertain modes allows the plant with uncertain natural frequency square and damping terms to be as shown in Fig. 1 with the following definitions:

lbegin equation

Delta $A_{-}\{L W\}=\mid$ Delta $A_{-}\{L D\}=$ E_ $\{2 k\},-\backslash ; ;\}$

Delta $A_{-}\{R W\}=-10 \operatorname{mega}^{\wedge} 2 \mathrm{E}^{\wedge} \mathrm{T} \_\{2 \mathrm{k}-1\}$, $1 ; i ;$

Delta A_ $\{R D\}=-\{$ lcal D $\}$ lOmega $\mathrm{E}^{\wedge} \mathrm{T}_{-}\{2 \overline{\mathrm{k}}\}$,

lend equation

lbegin equation

IOmega $=\{$ lrm diag $\}[$ lomega_ $\{k(i)\}], i ; j ;$;

\author{
$\{$ lcal D $\}=\{\mathrm{lrm} \operatorname{diag}\}[2$ lzeta_\{k(i) $\}]$, \\ ;; ; lforall $i=1,2$, Udots, $m, ~ i ; i ;$ \\ lend $\{$ equation \\ begin equation \\ $E \_\{2 k\}=\left[e_{-}\{2 k(1)\} ; ; j e \_\{2 k(2)\}\right) ;$; \\ $\mid c$ dots $; ;$ e_ $\{2 \mathrm{k}(\mathrm{m})\}]$ \\ lend $\{$ equation

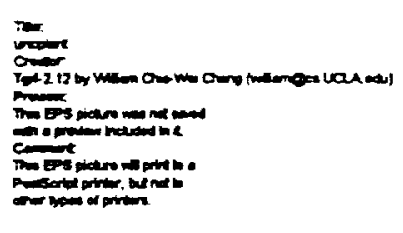 \\ Figure 1. Plant with Uncertain Modal \\ Damping \\ and Frequency Square Terms
}

Fig. 2 illustrates the generalized plant for robust control design. In addition to the uncertainty in the modal frequency square terms, an additive uncertainty is included to represent model error outside the control bandwidth. This type uncertainty model forces the controller to gain stabilize the high frequency modes that were truncated from the evaluation model. Additional inputs for the robust control design generalized plant include inputs associated with the additive uncertainty, wa, and the modal frequency uncertainty, wm.

Additional outputs include those associated with the additive uncertainty, za, and the modal frequency uncertainty, $\mathrm{zm}$. 


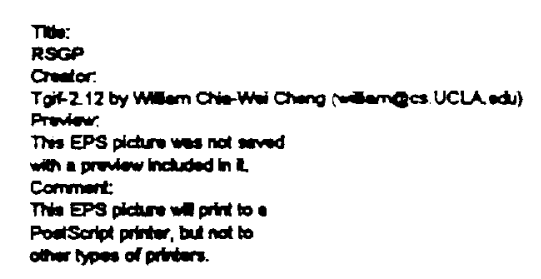

Figure 2. Generalized Plant for Robust Control Design

\section{Controller Design Approaches}

$\mathrm{H}_{2}$ methods are often used when designing control systems to reduce the vibration response of a flexible structure. While $\mathrm{H}_{2}$ design gives good nominal performance, the controllers are highly tuned to the design model and errors in the design model are not accounted for, typically inducing instability at higher levels of control authority. As a result, the actual performance achievable is limited with $\mathrm{H}_{2}$ designs. To achieve high levels of performance in the actual system, robustness to model errors must be taken into account in the design process. In this section, a brief introduction to $\mathrm{H}_{2}, \mathrm{H}_{\infty}, \mu$ synthesis, and mixed $\mathrm{H}_{2} / \mu$ control design is given. In the following section, these methods will be used to design controllers for the benchmark structural control problem and to demonstrate the significance of designing for nominal performance and robust stability. For more details on the theoretical basis of the control design methods used in this paper, see Refs. Vref\{Sweriduk\} and tref $\{$ Calise $\}$.
Design for Nominal Performance The generalized plant may be written in state space form as

begin $\{$ eqnarray $\}$

$\mid \operatorname{dot}\{x\} \&=\& A x+B \_1 w+B \_2 u \|$

$z \&=\& C_{-} 1 x+D_{-}\{12\}$ u $\|$

$y \&=\& C_{-} 2 x+D_{-}\{21\} \mathrm{w}+\mathrm{D}_{-}\{22\} \mathrm{u}$

lend $\{$ eqnarray $\}$

where $\$ x$ lin $\backslash R e^{\wedge} n \$$ is the state vector, $\$ w$ lin $\backslash R e^{\wedge}\{n w\} \$$ is the disturbance vector, $\$ u$ lin $\left\langle\operatorname{Re}^{\wedge}\{\mathrm{nu}\} \mathbf{S}\right.$ is the control vector, $\$ z$ lin $\backslash \operatorname{Re}^{\wedge}\{n z\} \$$ is the performance vector, and $\$ y$ lin $\backslash \operatorname{Re}^{\wedge}$ \{ny $\}$ S is the measurement vector. The $\mathrm{H}_{2}$ optimization problem is to find a stabilizing controller that minimizes the $\mathrm{H}_{2}$ norm of the closed loop system from disturbance inputs $\$ w \$$ to performance outputs \$z\$, denoted \$T_\{zw $\}$ S. The closed loop system may also be written as the linear fractional transformation (LFT) shown in Fig. 3. Another approach to design for nominal performance employs the $\mathbf{H}_{\infty}$ norm, which can be interpreted as the gain of the system and is the worst-case amplification over all inputs $w(t)$ of unit energy. From a frequency domain perspective, the $\mathrm{H}_{\infty}$ norm is defined as the maximum singular value of $\mathrm{T}(\mathrm{s})$ over all frequencies, i.e.

lbegin $\{$ equation

Iparallel T_\{zw $\}$ \parallel_linfty $=$ Isup_lomega \\{\bar|sigma

$\left.\left(T_{-}\{z w\}(j l o m e g a)\right) \backslash\right\}$

lend equation\}

$\mathrm{H}_{\infty}$ control design theory, based on Refs. Vref\{Francis $\}$ and $\operatorname{tref}\{D G K F\}$, involves 
defining (possibly frequency dependent) weights on the inputs and outputs such that the performance objectives are satisfied by minimizing \$lparallel T_\{zw Iparallel_linfty\$. Because the $\mathrm{H}_{\infty}$ norm is defined with respect to the peak magnitude of the transfer matrix frequency response and the $\mathrm{H}_{2}$ norm is defined by an integral square quantity (in time or frequency by Parseval's Theorem), the respective closed loop systems may have considerably different characteristics. Depending on the performance objectives, one design procedure may be preferable to the other. With regard to $\mathrm{rms}$ performance specifications, $\mathrm{H}_{2}$ design typically yields better nominal performance. The significant benefit of $\mathrm{H}_{\infty}$ theory is that robustness to model errors is explicitly factored into the design process.

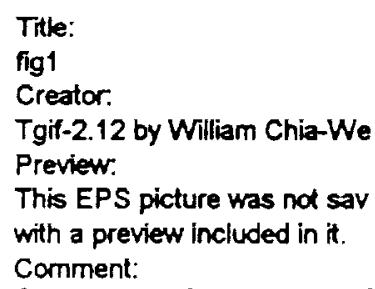

Figure 3. Linear Fractional Transformation of Closed

Loop

\section{Design for Robust Stability}

In addition to nominal performance, robust stability is an important design consideration. Robust stability requires the closed loop system to remain stable for bounded model errors. The uncertainty may be modeled in many forms such as multiplicative, inverse multiplicative, additive, parametric, etc. and may be located at various points in the loop. Recall that in an earlier section a model was presented for the benchmark problem with parametric and additive uncertainty modeled. By absorbing all of the scalings and weights into the plant $P$, the robust stability problem may be formulated as the LFT in Fig. 4 Vref $\{$ fig:RSLFT\}. The uncertainties are scaled so that \$Delta_ldelta\$ is the set of all stable perturbations such that Slparallel Delta Iparallel_linfty Veq Ideltas. Assuming that $\mathrm{K}(\mathrm{s})$ internally stabilizes the closed loop for $\$ \backslash D$ elta $=0 \$$, then a sufficient condition for robust stability for all plants in the set formed by \$Delta in Delta_Idelta\$ is \$ $\$$ rm that ${ }^{\wedge}\{$ Iref $\{$ Maciejowski\}, $\operatorname{tref}\{$ Morari\}\}\$

Jbegin \{equation\}

Iparallel T_ $\{z w\}(K) \backslash$ parallel_linfty Veq lfrac $\{1\}\{$ ldelta $\}$

Vabel\{eq:RS $\}$ lend \{equation\}

Thus like the nominal performance problem, robust stability is provided by minimizing the norm of a particular transfer function.

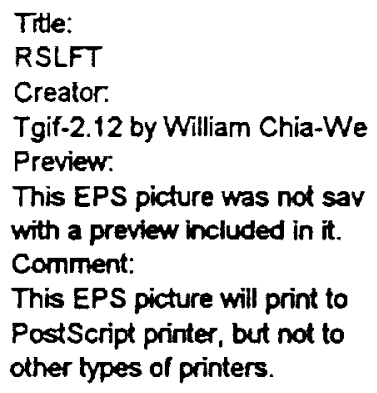

Figure 4. LFT for Robust Stability Analysis

\section{Design for Robust Performance}

It is the ability to formulate the performance problem as a robust stability problem that enables robust performance controller design in the $\mathrm{H}_{\infty}$ setting. Consider the uncertain plant in Fig. 2 with inputs and 
outputs defined for performance and an uncertainty model. The plant is recast as an LFT in Fig. 5 where

lbegin $\{$ equation

w_l=Vleft[begin $\{$ array $\}\{c\}$ w_m $\|$ w_a lend \{array\} Vright] i;

w_2 $=$ Veft $\llbracket$ begin $\{$ array $\}\{c\}$ Vrm noise $\} \|$ Iddot $\{x\}$ g lend $\{$ array $\}$ lright $]$ i;;

z_1=Veft[begin $\{$ array $\}\{c\}$ z_m $\| z_{-}$a lend \{array\} lright] $;$;

z_2=Vleft[begin $\{$ array $\}\{c\} z$ z $\| z u$ lend \{array\} lright] 11

lend equation

The conditions for robust performance are:

begin $\{$ enumerate

litem robust stability (Eq. $\operatorname{Vef}\{$ eq:RS\}), and

litem performance maintained for all \$UDelta lin Welta_Idelta\$

lend enumerate

Closing the loop from $\$ z 2 \$$ to $\$ w 2 \$$ through a fictitious uncertainty block \$LDelta p\$ recasts the robust performance problem as a robust stability problem, shown in Fig. 5 where the blocks are scaled to one.

A sufficient condition for robust performance is that
Iparallel $\mathrm{T}(\mathrm{K})$ |parallel_linfty $<1$

Vabel $\{$ eq:scRP $\}$

lend equation

Define $\$\{$ lunderlinelDelta\} $1 \$$ to be the set of all stable, bounded, unstructured perturbations \$\{lunderlinelDelta\}\$ such that $\$$ iparallel \{lunderlinelDelta\} |parallel_linfty $<1 \$$. When $\$$ lunderlinelDelta\} lin \{lunderlinelDelta\}_1\$, Eq. Vef $\{$ eq:scRP\} is necessary and sufficient to ensure robust stability. Designing for robust performance using \$Delta $\mathrm{p} \$$ as in Fig. 5 introduces a block diagonal structure to \$\{lunderlinelDelta\}\$ which results in Eq. Iref $\{$ eq:scRP $\}$ being only sufficient and possibly overly conservative. This

conservatism is relaxed in the $\mu$-analysis and $\mu$-synthesis $\$ V \mathrm{rm}$ procedures $\wedge\{$ Vref $\{$ Doyl $\}$, Iref $\{$ Doy2\}, Lref\{DoyChu\}\}S by accounting for the block diagonal structure in $\$$ \{lunderlinelDelta\}\$.

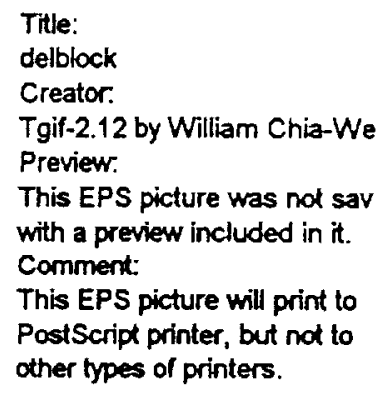

Figure 5. LFT for Robust Performance Design

The structured singular value is used to define the $\mu$-measure, which although not a norm, is denoted lbegin equation

lbegin\{equation\} 
iparallel $T$ (jlomega) $\backslash$ parallel_\mu =

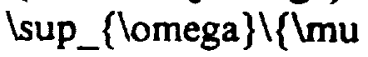

(T_\{zw $\}($ jlomega $)) \backslash\}$

lend equation\}

Hence the sufficient condition for robust performance in Eq. Vref $\{$ eq:scRP\} becomes the necessary and sufficient condition lbegin \{equation

\parallel T(jlomega) \parallel_ımu $<1$ Vabel $\{$ eq:ncRP $\}$

lend equation

Although the structured singular value cannot be directly computed, an upper bound

can be computed as

lbegin \{equation

$\operatorname{lmu}(T)=\operatorname{linf}_{-}\{\mathrm{D}\} \backslash \backslash$ barlsigma(DTD $\mathrm{D}^{\wedge}\{-$ 1\})।\}

Vabel $\{$ eq:mubound $\}$

lend equation\}

where $\$ D=\{\backslash r m$ diag $\}\left[d \_j \_j\right] \$$ has the same structure

as \$D\$ and \$d j\$ are scalar, positive, real functions of frequency. An

iterative scheme is used to solve this optimization problem. In the

first step, an $\mathrm{H}_{\infty}$ controller is designed and in the second step, the

\$D\$-scales are optimized for this controller in accordance with Eq.

Iref $\{$ eq:mubound\}. In the next iteration, these \$D\$-scales are incorporated into the generalized plant and the control design is repeated, followed again by

\$D\$-scaling. This iterative process continues until the upper bound in Eq. Iref\{eq:mubound\} cannot be reduced significantly.

Design for Robust Stability and Nominal Performance

Although $\mu$-synthesis provides stability and performance in the presence of model errors, the performance is defined by an $\mathrm{H}_{\infty}$-norm measure which may yield poor $\mathrm{H}_{2}$ performance. The mixed $\mathrm{H}_{2} / \mathrm{H}_{\infty}$ design procedure has been developed to provide robust stability and nominal $\left(\mathrm{H}_{2}\right)$ performance by minimizing the $\mathrm{H}_{2}$ norm for one set inputs/outputs while satisfying an $\mathrm{H}_{\infty}$-norm overbound for another set of inputs/outputs. With reference to Fig. 5, the objective is to satisfy

lbegin \{equation\}

Imin_K parallel T_\{z2w2\} $\mid$ parallel_2

lend (equation\}

subject to

lbegin \{equation\}

Iparallel T_\{zlw1\} \parallel_linfty < Igamma

lend \{equation\}

This problem has been solved for controllers of fixed \$ $\mathrm{trm}$ dimension^\{lref\{Sweriduk\}, Vref\{Ridgely\}\}\$ with a numerical homotopy algorithm for the formulation of Ref. 7 given in Ref. 17. The homotopy algorithm that solves the necessary conditions for a fixed order mixed $\mathrm{H}_{2} / \mathrm{H}_{\infty}$ (or $\mu$ ) controller is a two parameter iterative scheme which effectively trades between robust stability and nominal performance by varying the overbound on the $\mathrm{H}_{\infty}$-norm, \$lgamma\$, and the weight on the $\mathrm{H}_{2}$ cost, \$Vambda\$. 
For a given \$lgamma, ; VambdaS is increased until the $\mathrm{H}_{\infty}$-norm constraint becomes an active, equality constraint (at which point the $\mathrm{H}_{2}$ norm can no longer be reduced) or until the $\mathrm{H}_{2}$ norm ceases to decrease. The set of controllers where the $\mathrm{H}_{\infty}$-norm is equal to the overbound are called the boundary solutions, the set of which provides an explicit trade between nominal performance and robust stability. By incorporating the $\mathrm{D}$-scales from $\mu$ synthesis into the

$\mathrm{H}_{\infty}$ subproblem, the structure of the uncertainty block may be accounted for, resulting in a fixed order mixed $\mathrm{H}_{2} / \mu$ design procedure. The next section presents a brief overview of the numerical algorithm used for fixed order mixed norm controller synthesis.

\section{Controller Design Results}

This section presents a comparison of the design approaches for nominal performance $\left(\mathrm{H}_{2}\right.$ ), robust performance ( $\mu$-synthesis), and nominal performance/robust stability (mixed $\mathrm{H}_{2} / \mu$ ) for the benchmark structural control problem. For evaluating the nominal performance of these designs, performance is defined by the rms response of the three relative floor displacements, $\mathrm{Vz}$, and the rms control effort, $\mathrm{Vu}$.

With reference to Fig. Iref $\{$ fig:H2GP $\}$ for the $\mathrm{H}_{2}$ nominal performance design, the disturbance input and performance output vectors are begin \{equation\}

$w=V$ left[begin $\{$ array $\}\{c\}\{$ lrm noise $\} 11$ lddot $\{\mathbf{x}\} \_$lend $\{$array $\}$Vright $\} ; ;$

$z=V$ left[begin $\{$ array $\}\{c\} z \_p \| z \_u$

lend \{array\} lright] 11

lend equation\}

The design parameters are defined as follows: the control weight, $\mathrm{Wu}=$ Slsqrt $\{\rho\} S$, the weight on relative displacement of each floor, $W p=25$, the sensor noise intensity, $\mathrm{Kn}=0.001$, and the intensity of the ground disturbance, $\mathbf{K d}=$ 0.0017 (chosen to match the dc intensity of the Kanai-Tajimi (K-T) spectrum). Control authority was varied in the design process using the scalar $\rho$.

For the $\mu$-synthesis design, Fig. 2 Vref $\{$ fig:RSGP\} is used where the uncertainty model included $51 \%$ uncertainty for the natural frequency square error $(\mathrm{Wm}$ $=\$$ isqrt $\{0.005\} \$$ ) and the additive uncertainty weighting function is given by begin equation\}

W_ $\{$ additive $\}=6.4$

Ufac $\left\{(s+5)^{\wedge} 3\right\}\left\{(s+200)^{\wedge} 3\right\}$ Vabel $\{$ eq:wadd $\}$

lend equation

In order to balance the plant for improved numerical results, the additive uncertainty model is realized as the frequency dependent term, Wadd, and the constant gain term, Kadd, as indicated in Fig. 2 vef\{fig:RSGP\}. The uncertainty block has the structure

begin \{equation\} 
Delta $=\operatorname{Vef}[$ begin $\{$ array $\}\{c \mathrm{ccc} c\}$

|delta_1 \& \& \& \& II

\& Idelta_2 \& \& 0 \& 11

\& \& Idelta_3 \& \& II

\& 0 \& \& Delta_ 4 \& II

\& \& \& \& Welta_p

lend \{array\} light]

lend equation\}

with SLelta_4 in $C^{\wedge}\{3 \times 1\}$; $\{\mathrm{rm}$ and $\}$; Delta_ $p$ lin $\bar{C}^{\wedge}\{4 \times 4\} S$.

For the $\mu$-synthesis design, the corresponding disturbance and performance vectors are

lbegin \{equation\}

w=Veft[begin $\{$ array $\}\{c\}$ w_m $\|$ w_a $\|$

$\{$ Irm noise $\} \| I$ ldot $\{x\} \_$lend $\{$array $\}$lright $]$ ;i;

$z=$ Veft $\left[\right.$ begin $\{$ array $\}\{c\} z_{-} m\left\|z_{-} a\right\| z_{-} u \|$ $z \_p$ lend $\{$ array\} $\}$ right $] \|$

lend equation $\}$

A set of $\mu$ controllers of varying control authority

was designed by fixing $W p$ and varying $\rho$ to achieve good nominal

performance. In order to make a consistent comparison of control approaches from a robustness perspective, each controller was

designed to achieve a $\mu$

measure of one so that achievable performance given a fixed level of robustness could be evaluated. First order \$D\$-scales were used for each $\mu$ controller design, resulting in $\mu$ controllers with 19 states computed using the MATLAB $\mu$ Analysis and Synthesis Toolbox. ${ }^{18}$

Finally, a set of mixed $\mathrm{H}_{2} / \mu$ controllers were designed with fixed controller dimension of 6 th order using the homotopy algorithm of Ref. 17. In order to trade between nominal performance and robust stability, the $\mathrm{H}_{2}$ subproblem is defined for nominal performance as above and the $\mu$ subproblem accounts for the additive and parametric uncertainty models. The problems are defined by the inputs and outputs

lbegin \{equation\}

$\mathrm{w}_{-} \mathrm{l}=$ Ueft[lbegin $\{$ array $\}\{\mathrm{c}\} \mathrm{w}_{-} \mathrm{m} \| \mathrm{w}{ }_{-} \mathrm{a}$ lend \{array\} lright] $\ggg ;$

$z_{-} 1=$ Veft $\mid$ begin $\{$ array $\}\{c\} z_{-} m \| z_{-} a$ lend \{array\} lright] $; i ; ;$

w_2 $=$ Veft[begin $\{$ array $\}\{c\}\{\mathrm{lm}$ noise $\} \|$ Iddot $\{x\}$ g lend array $\}$ \right } ] \text { ;; }

z_2=Ueft[begin $\{$ array $\}\{c\} z \_p \| z u$ lend \{array\} lright] 11

lend equation\}

and the \$D\$-scales for the $\mu$ subproblem are obtained from \$D-K\$ iterations for \$T_\{zlw1\}\$.

Fig. 6 presents the rms nominal performance curves for each control design method. The robust control designs are for the baseline uncertainty model (which has $5 \%$ uncertainty in the natural frequency square parameters and the additive uncertainty). The costs are computed with the K-T spectrum input. $\mathrm{H}_{2}$ design costs are 
computed for both the design and evaluation models to illustrate the limitation on achievable performance due to model error. Although the cost curve evaluated with the design model extends to high control authority levels, the maximum performance with the evaluation model is obtained at $\rho=$ 15.63. The loop closed with the $\mathrm{H}_{2}$ controllers and the evaluation model are unstable for smaller values of $\rho$. This cost comparison also indicates that for control authority levels lower than the instability level, the actual performance is almost identical to the design model performance.

\section{manim \\ $\operatorname{mon}_{0 \rightarrow 0}$ morangene. mosencines}

Figure 6. RMS Performance Comparisons

Fig. 6 also indicates the loss of $\mathrm{rms}$ performance that is incurred in exchange for robust performance. As a basis for comparison the set of $\mu$ designs is evaluated in terms of rms performance. $A$ substantial gap in performance exists between the $\mathrm{H}_{2}$ and $\mu$ designs since the $\mu$ designs achieve a given level of output performance at a higher control cost than the $\mathrm{H}_{2}$ designs. However, the mixed $\mathrm{H}_{2} / \mathrm{H}_{\infty}$ designs effectively recover the rms performance of the $\mathrm{H}_{2}$ designs while providing the same level of robust stability as the $\mu$ designs. The mixed $\mathrm{H}_{2} / \mu$ design procedure provides performance comparable to $\mathrm{H}_{2}$ design while overcoming the major shortcoming of $\mathrm{H}_{2}$ design, namely a lack of stability robustness.

The impact of uncertainty on performance in the mixed norm design setting is evident in Fig. 7 where a set of mixed norm designs are evaluated with $10 \%$ and $20 \%$ parametric uncertainty in addition to the baseline 5\% parametric uncertainty. As the level of robustness increases, performance in sacrificed as indicated by the upward shift in the performance curve. A cursory comparison of Figs. 6 and 7 indicates that the mixed norm controllers designed for $10 \%$ and $20 \%$ parametric uncertainty yield comparable performance to the $\mu$ controllers designed for $5 \%$ uncertainty. Hence the mixed norm designs provide more robust stability for a given level of performance than the $\mu$ controllers. Note that these comparisons are for nominal performance and may not hold for robust performance. In these analyses, the additive uncertainty is held fixed since it is defined with respect to the model and serves only to force the controller to roll off and gain stabilize the high frequency unmodeled dynamics. 


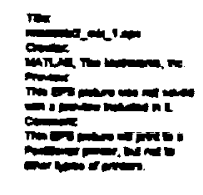

Figure 7 Impact of Uncertainty Level on

\section{RMS Performance}

Robust stability of each design is evaluated using mixed $\mu$ analysis where the parametric uncertainty is considered real and the additive uncertainty complex. As a result, the mixed Im measure is a less conservative measure of robust stability.

Fig. 8

plots the $\mathrm{lm}$ measure for the set of $\mathrm{H} 2$ controllers for varying authority levels as a function of parametric uncertainty level. This plot should be interpreted as indicating the magnitude of perturbation

required to destabilize the closed loop.

From Eq. Iref $\{$ eq:RS\}, a $\mu$ measure $\$<1 \$$ indicates robust stability is guaranteed for all plants in the uncertain set. For a controller associated with a $\mu$ measure of

\$Ibeta\$, the system will be unstable for \$iparallel Delta Iparallel_linfty lgeq Ifrac $\{1\}\{$ (beta\}\$. The $\mathrm{H}_{2}$ designs are robust with respect to the uncertainty model only for very low authority controllers.

Fig. 8 illustrates the well known property of $\mathrm{H}_{2}$

controllers that as control authority increases, the sensitivity (in terms of stability) to model error increases. This figure also indicates that the Im measure is relatively insensitive to different levels of parametric uncertainty at high control authority levels which indicates that the additive uncertainty dominates the stability analysis. Only at low authority levels are the $\mathrm{H}_{2}$ designs sensitive to parametric uncertainty. Since control bandwidth is proportional to the authority level for these $\mathrm{H}_{2}$ designs, the higher authority controllers interact with and destabilize the unmodeled modes.

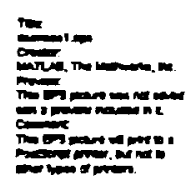

Figure 8. Robust Stability Analysis of $\mathrm{H}_{2}$ (top) Controllers and Mixed Norm Designs Performed for $5 \%$ Uncertainty (bottom) 


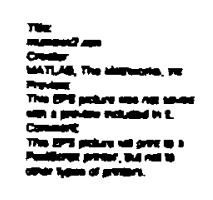

Figure 9. Robust Stability Analysis of

Mixed Norm Designs Performed for $10 \%$ (top) and 20\% Uncertainty (bottom)

Robust stability analyses of the mixed norm designs for $5 \%, 10 \%$, and $20 \%$ parametric uncertainty are shown in Figs. 8 and 9. For the $\$ 51 \% \$$ uncertainty design, an $\mathrm{H}_{\infty}$ overbound of one was achieved.

Although robust stability is not guaranteed for levels of uncertainty above $\$ 51 \% \$$, the $\mathrm{lm}$ measure for $\$ 251 \% \$$ parametric uncertainty is less than two, which is roughly three times better than the $\mathrm{H}_{2}$ designs. It is also interesting to note that the $\mathrm{lm}$ measure for the mixed norm design is sensitive to differences in parametric uncertainty and is relatively insensitive to the control authority, which is opposite the characteristic of the $\mathrm{H}_{2}$ designs. As a matter of fact, the $\mathrm{Im}$ measure decreases slightly with control authority for the mixed norm designs. Somewhat different behavior is observed with the mixed norm designs for $\$ 101 \% \$$ and $\$ 201 \% \$$ parametric uncertainty. The mixed norm design set for $\$ 101 \% \$$ parametric uncertainty used an $\mathrm{H}_{\infty}$ overbound of $\$ 1.3 \$$, so robust stability is not fully guaranteed for $\$ 101 \% \$$ variations in the uncertain natural frequency. From Fig. 9, the peak $\mathrm{lm}$ measure for $\$ 101 \% \$$ parameter uncertainty

is $\$ 1.26 \$$. Similarly for the mixed norm design with $\$ 201 \% \$$ parametric uncertainty, an $\mathrm{H}_{\infty}$ overbound of $\$ 2.1 S$ was used and the peak $\mathrm{lm}$ measure is $\$ 1.75 \$$. These two designs have a characteristic behavior more similar to the $\mathrm{H}_{2}$ designs in that the $\mathrm{Im}$ measure is more

sensitive to control authority than parametric uncertainty level.

However, the variation with control authority is significantly less than the $\mathrm{H}_{2}$ designs.

\section{Conclusions}

This paper has presented a comparison of $\mathrm{H}_{2}, \mu$-synthesis, and mixed $\mathrm{H}_{2} / \mu$ control design for a structural benchmark control problem with an emphasis on the issues of robust stability and nominal performance. A particular uncertainty model was employed which accounted for uncertainty in the natural frequency of each mode in the control bandwidth and an additive uncertainty to provide stability in the presence of high frequency unmodeled modes. It has been shown that although $\mathrm{H}_{2}$ design yields good nominal performance, the designs have poor stability characteristics with respect to errors in the design model. $\mu$-synthesis designs provide robust stability, but tend to sacrifice performance for robust stability and result in controllers with higher control authority than the $\mathrm{H}_{2}$ designsfor a given level of performance. A fixed order mixed $\mathrm{H}_{2} / \mu$ design approach was introduced which provides the same robust stability guarantees 
as the $\mu$-synthesis designs while almost fully recovering the $\mathrm{H}_{2}$ nominal performance levels. Accounting for the model errors also permits higher levels of control authority for which the $\mathrm{H}_{2}$ designs are destabilizing with the evaluation model. This mixed norm design approach was demonstrated to be an effective means for designing $\mathrm{H}_{2}$ controllers with robust stability for the benchmark structural control problem.

\section{References}

1. G. W. Housner and A. F. Masri, Int. Workshop on Struct. Control, University of Southern Califomia Press, Los Angeles, CA, 1993.

2. W. E. Schmitendorf, F. Jabbari, and J. N. Yang, "Robust control techniques for buildings under earthquake excitations," Earthquake eng. struct. dyn., 23, 539-552, (1994).

3. J.N. Yang, J. C. Wu, A. M. Reinhorn, M. Riley, W. E. Schmitendorf, and F. Jabbari, "Experimental verifications of $\mathrm{H}_{\infty}$ and sliding mode control for seismic excited buildings," ASCE J. struct. eng., $122,69-75$.

4. I. E. Kose, W. E. Schmitendorf, F. Jabbari, and I. N. Yang, (1996), "Active seismic response control using static output feedback," $A S C E J$. of Eng. Mech., 122, 651-659 (1996).

5. B. F. Spencer, Jr., S. Dyke, and H. Deoskar, "Benchmark problems in structural control," Proc. 1997 ASCE
Structures Congress, April 13-16, Portland, OR, 1997.

6. G. J. Balas, and P. M. Young, "Control design for variations in structural natural frequencies," Journal of Guidance, Control, and Dynamics, 18, MarchApril, 325-332 (1995).

7. G. D. Sweriduk and A. J. Calise, "A differential game approach to the mixed $\mathrm{H}_{2} / \mathrm{H}_{\infty}$ problem," AIAA Paper 94-3660CP, August 1994.

8. A. J. Calise and G. D. Sweriduk, "Active attenuation of building structural response using robust control," $A S C E J$. Engr. Mechs., accepted subject to minor revisions.

9. B. A. Frances, A Course in $\mathrm{H}_{\infty}$ Control Theory, Springer-Verlag, Berlin, 1987.

10. J. C. Doyle, K. Glover, P. Khargonekar, and B. A. Francis, "State-space solutions to standard $\mathrm{H}_{2}$ and $\mathrm{H}_{\infty}$ control problems," IEEE Transactions on Automatic Control, 34, 831-847 (1989).

11. J. M. Maciejowski, Multivariable Feedback Design, Addison Wesley, 1989.

12. M. Morari and E. Zafiriou, Robust Process Control, Prentice Hall, 1989.

13. J. C. Doyle, "Analysis of feedback systems with structured uncertainties," Proc. IEE-D 129, 242-250 (1982).

14. J. C. Doyle, "Lecture Notes on Advances in Multivariable Control," ONR/Honeywell Workshop on Advances in Multivariable Control, Minneapolis, MN, October, 1984.

15. J. C. Doyle and C. C. Chu, "Robust control of multivariable and large scale systems," Final Technical Report for AFOSR, Contract No. F49620-84-C0088, March 1986. 
16. D. B. Ridgely, R. A. Canfield, D. E. Walker, and L. D. Smith, "The fixed order mixed $\mathrm{H}_{2} / \mathrm{H}_{\infty}$ control problem: development and numerical solution," The International Journal of Robust and Nonlinear Control (submitted for publication).

17. M. S. Whorton, H. Buschek, and A. J. Calise, "Homotopy algorithm for fixed order mixed $\mathrm{H}_{2} / \mathrm{H}_{\infty}$ design", Journal of Guidance, Control, and Dynamics, 19, 1262-1269 (1996).

18. G. J. Balas, J. C. Doyle, K. Glover, A. Packard, and $R$ Smith, $\mu$-Analysis and Synthesis TOOLBOX The Mathworks, 1993. 